\title{
Neurotrophin expression and laryngeal muscle pathophysiology following recurrent laryngeal nerve transection
}

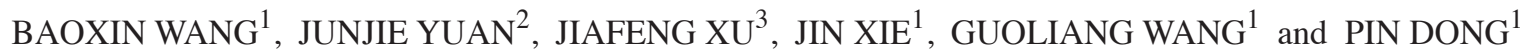 \\ ${ }^{1}$ Department of Otolaryngology, Head and Neck Surgery, Shanghai General Hospital, \\ Shanghai Jiao Tong University School of Medicine, Shanghai 201620; ${ }^{2}$ Department of Orthopedics,
} Shanghai Fengxian District Central Hospital, Shanghai Jiao Tong University Affiliated Sixth People's Hospital South Campus, Shanghai 200011; ${ }^{3}$ School of Economics and Finance, Shanghai International Studies University, Shanghai 200083, P.R. China

Received March 25, 2015; Accepted November 19, 2015

DOI: $10.3892 / \mathrm{mmr} .2015 .4684$

\begin{abstract}
Laryngeal palsy often occurs as a result of recurrent laryngeal or vagal nerve injury during oncological surgery of the head and neck, affecting quality of life and increasing economic burden. Reinnervation following recurrent laryngeal nerve (RLN) injury is difficult despite development of techniques, such as neural anastomosis, nerve grafting and creation of a laryngeal muscle pedicle. In the present study, due to the limited availability of human nerve tissue for research, a rat model was used to investigate neurotrophin expression and laryngeal muscle pathophysiology in RLN injury. Twenty-five male Sprague-Dawley rats underwent right RLN transection with the excision of a 5-mm segment. Vocal fold movements, vocalization, histology and immunostaining were evaluated at different time-points (3, 6, 10 and 16 weeks). Although vocalization was restored, movement of the vocal fold failed to return to normal levels following RLN injury. The expression of brain-derived neurotrophic factor and glial cell line-derived neurotrophic factor differed in the thyroarytenoid (TA) and posterior cricoarytenoid muscles. The number of axons did not increase to baseline levels over time. Furthermore, normal muscle function was unlikely with spontaneous reinnervation. During regeneration following RLN injury, differences in the expression levels of neurotrophic factors may have resulted in preferential reinnervation of the TA muscles. Data from the present study indicated that neurotrophic factors may be applied for restoring the function of the laryngeal nerve following recurrent injury.
\end{abstract}

Correspondence to: Dr Pin Dong, Department of Otolaryngology, Head and Neck Surgery, Shanghai General Hospital, Shanghai Jiao Tong University School of Medicine, 100 Haining Road, Shanghai 201620, P.R. China

E-mail: dongpin64@aliyun.com

Key words: regeneration, nerve injury, brain-derived neurotrophic factor, glial cell line-derived neurotrophic factor

\section{Introduction}

Peripheral nerves enable communication between the brain and muscles, skin and organs, and peripheral nerve injury often results in the loss of sensory or motor function $(1,2)$. The majority of the functions of the larynx depend on healthy vocal fold motion, which in turn relies on an intact recurrent laryngeal nerve (RLN) (3). Laryngeal palsy often occurs as a result of recurrent laryngeal or vagal nerve injury during oncological surgery of the head and neck (4). Despite a standardized surgical approach, it is difficult to locate the RLN or protect it when scar tissue, inflammatory changes, malignancies of the thyroid, or anatomical variations in the RLN are present (5). During surgery, the RLN may be injured as a result of stretching, compression, cutting or cauterizing (6). Although the injured RLN regenerates, even across a gap $(7,8)$, absolute normal function is not recovered due to laryngeal muscle atrophy, motor neuron loss, decreased motor fiber density, or synkinesis (9-12). Furthermore, the regeneration potential of the RLN is associated with the severity of the injury (13).

The symptoms and signs of laryngeal paralysis vary (14). Although a number of patients with unilateral vocal fold paralysis are completely asymptomatic, others may experience disabling symptoms, such as serious hoarseness, dysphagia and dysphonia (15). In addition to affecting the quality of life, RLN injury may impose psychosocial and economic burdens (16). The vocal folds are fragile structures, and even a slight injury may result in extreme complications. Early intervention is important for complete recovery and preservation of laryngeal function (17). Reinnervation techniques, such as neural anastomosis, nerve grafting and creation of a laryngeal muscle pedicle have been reported, however, they have had little impact and are not widely accepted as treatment methods (18).

Spontaneous reinnervation is normal following RLN injury (19-22). In patients, RLN reinnervation improves muscle tone and enables vocal fold medicalization (23-25). However, as it cannot restore motion, RLN reinnervation is not beneficial for patients with unilateral paralysis (19).

Although the rat larynx is small, it has been used as a model for investigating RLN injury and recovery (6). In previous studies, peripheral nerve injury models involved crushing, 
transecting, cutting, compression, stretching and cauterizing the nerve (26-39). The present study selected the transection model, as it utilizes a simple and reproducible technique, and is ideal for analyzing the characteristics of spontaneous reinnervation.

Neurotrophic factors are a group of proteins that have been demonstrated to prevent motor neuron loss, and promote differentiation and reinnervation (40-45). Neurotrophic factors are observed in neural tissue and in skeletal muscles, where they provide a retrograde trophic influence on innervating motor neurons (45). Previous studies determined the effects of various neurotrophic factors, such as brain-derived neurotrophic factor (BDNF), ciliary neurotrophic factor, neurotrophin-4, and nerve growth factor, in RLN injury $(4,17,19,22,46-51)$; treatment with neurotrophins may stimulate nerve regeneration (48).

The aim of the present study was to characterize the features of spontaneous reinnervation in rats following RLN injury. Therefore, based on previous studies, two neurotrophic factors, BDNF and glial cell line-derived neurotrophic factor (GDNF) were selected to investigate their expression levels following nerve injury.

\section{Materials and methods}

Experimental animals. The present study was conducted on 25 male Sprague-Dawley rats (weight, 320-350 g) purchased from the Shanghai SLAC Laboratory Animal Co., Ltd. (Shanghai, China). The rats were maintained in the animal laboratory of the Shanghai Jiao Tong University affiliated Shanghai First People's Hospital (Shanghai, China) and all surgical procedures were performed in the animal operating room. The experiment was approved in accordance with the Institutional Animal Care and Use guidelines of Shanghai Jiao Tong University by the Animal Experimental Committee of First People's Hospital affiliated with the Shanghai Jiao Tong University of Medicine. The animals were treated humanely. All rats were maintained under standardized laboratory conditions in groups of 5 rats, with food and water available ad libitum. The humidity was $60 \%$ and the air temperature was $25^{\circ} \mathrm{C}$, with a $12 \mathrm{~h}$ light/dark cycle.

Surgical procedure. Following intraperitoneal anesthesia with sodium pentobarbital (40 mg/kg body weight; Shanghai Yuanye Biotechnology Co., Ltd., Shanghai, China), telescopic video laryngoscopy (BF-1T160; Olympus Corporation, Tokyo, Japan) was performed to confirm normal motion of the vocal fold with breathing. Following removal of the hair on the neck, a vertical midline cervical incision was made with a sharp blade (Tongkang Medical Instrument Co., Ltd., Shanghai, China). To expose the tracheoesophageal groove, the salivary glands and infrahyoid muscles were reflected laterally using iris hooks (Tongkang Medical Instrument Co., Ltd.). Subsequently, the right RLN was carefully isolated from the tracheoesophageal groove. The animals were divided into two groups, with 5 rats/group. For the denervation group, the right RLN was resected at the level of the seventh tracheal ring, with the removal of a 5-mm segment. The muscle layers and skin were sutured separately. For the control group, the incision was sutured following identification of the right
RLN. Telescopic video laryngoscopy was repeated to confirm paralysis of the right vocal fold. On occasions where the right vocal fold exhibited movement, the rat was excluded from further investigation. An intraperitoneal injection of benzylpenicillin sodium $\left(10^{5} \mathrm{U} / \mathrm{kg}\right.$ body weight; North China Pharmaceutical Co., Ltd., Shijiazhuang, China) was administered to all rats to prevent infection. All the rats were monitored following surgery, fed a standard diet, and allowed to heal for $3,6,10$ or 16 weeks $(n=5$ for each group and time period).

Vocal fold movement. At 3, 6, 10 and 16 weeks, five rats with transection injuries were anesthetized again, as described above. Previous studies have demonstrated that the effects of anesthesia on vocal fold mobility are minimal $(52,53)$. Video laryngoscopy was performed to assess the vocal fold motion and glottis morphology during spontaneous breathing. The movements observed via transoral endoscopy were divided into five different categories ranging from grade 0 to 4 using the following criteria: grade 0 , no vocal fold movement; grade 1, slight vocal fold movement; grade 2, <50\% abduction of vocal fold; grade $3,>50 \%$ abduction of vocal fold; and grade 4, normal vocal fold movement. The final scores were obtained by two investigators who were blinded to the animal grouping and time since denervation. In case of disagreement, a third investigator's score was obtained. Normal movement of the vocal fold was measured using five rats and a double-blind assay was performed to prevent subjective bias.

Images were captured for measurement of the arytenoid cartilages (Fig. 1). The angles between the arytenoid cartilage structures were calculated (54) using images of maximal adduction and abduction (Fig. 2).

Measurement of vocalization. Animals were maintained in a quiet environment for one day prior to euthanasia. Vocalization lasting $20 \mathrm{sec}$ was recorded by stimulating the right hind limb using forceps every $4 \mathrm{~h}$ following surgery. The procedures were performed in an identical environment by the same investigator using the same equipment, crush pressure $(5 \mathrm{~N})$, and distance between the recorder and mouth $(10 \mathrm{~cm})$. A capture tool (Cool Edit Pro v2.1; Adobe Systems, Inc., San Jose, CA, USA) was used to record the sound fragments, followed by acoustic space analysis using Photoshop CC software, version 14.0 (Adobe Systems, Inc.). Calculations were performed as follows: Spectral area $(\%)=$ area of postoperative rats/area of control rats. Amplitude $(\%)=$ amplitude of postoperative rats/amplitude of control rats.

Histologic examination. Following euthanasia using sodium pentobarbital (120 mg/kg body weight), the larynx was excised. Under a dissecting microscope (SZ51-ILST-SET; Olympus Corporation), the thyroarytenoid (TA) and posterior cricoarytenoid (PCA) muscles were removed for immunostaining of BDNF and GDNF. The TA and PCA muscles were selected for the current study as the TA muscles are hypothesized to be the most affected laryngeal muscles following resection of the RLN, and the PCA muscle is the sole abductor muscle (55). At each time-point, six sections (7 $\mu \mathrm{m}$ thick) from each rat were prepared for staining. Sections were pretreated with $0.3 \% \mathrm{H}_{2} \mathrm{O}_{2}$ 
and preincubated in 5\% bovine serum albumin (Wuhan Boster Biological Technology, Co., Ltd., Hubei, China). The sections were then incubated with a rabbit monoclonal antibody against BDNF (dilution, 1:75; ab108319; Abcam, Cambridge, MA, USA) or a rabbit polyclonal antibody against GDNF (dilution, 1:100; sc-328; Santa Cruz Biotechnology, Inc., Dallas, TX, USA), overnight at $4^{\circ} \mathrm{C}$, followed by incubation for $15 \mathrm{~min}$ at $37^{\circ} \mathrm{C}$ with the horseradish peroxidase-conjugated secondary antibody (GK500711; Genetic Technology Co., Ltd., Shanghai, China) according to the manufacturer's instructions. Following incubation with 3,3'-diaminobenzidine, the sections were dehydrated, dried and coverslipped.

Measurement of immunostaining. Two observers blinded to the grouping examined each section separately using a morphometric workstation running Image Pro Plus 6.0 software (Media Cybernetics, Inc., Rockville, MD, USA). Following calibration of the optical density, the images were recorded at a final magnification of x100. The area, mean density and relative optical density of immunolabeling were obtained for each specimen. The microscope illumination was calibrated to ensure that the relative optical density values were within the linear response range.

The right RLN was harvested. The left nerve served as a control and was harvested at the same level. The RLN was isolated and fixed in $4 \%$ (vol/vol) formaldehyde for $48 \mathrm{~h}$, embedded in paraffin and $7-\mu \mathrm{m}$ sections were cut from each segment, and examined by modified Bielschowsky staining (56). The slices were placed in silver nitrate solution for impregnation for $30 \mathrm{~min}$ and restored using formaldehyde. Silver ammonia was added, which was followed by another restoration. Finally, the sections were toned with gold chloride and fixed by sodium thiosulfate (Shanghai Yuanye Biotechnology Co., Ltd.). As a result, the axons appeared black. For each time-point, six sections from each rat were prepared for imaging. The diameter and number of axons were calculated using Photoshop CC software, version 14.0 (Adobe Systems, Inc.), and the longest and shortest axons in addition to the mean axon diameter were analyzed.

Statistical analysis. Each outcome value was summarized as the mean \pm standard deviation. A one-way analysis of variance with Tukey's multiple comparison tests was used for statistical analysis of the measures of vocal fold movement and vocalization using SPSS version 20.0 (IBM SPSS, Armonk, NY, USA). The immunostaining and histological results were analyzed using the independent-samples t-test. $\mathrm{P}<0.05$ was considered to indicate a statistically significant difference.

\section{Results}

Animal survival and recovery. All animals survived until the predetermined endpoint. All incisions healed well without infection. Nerve ends were surrounded by scar adhesions, with no significant bulges.

Effect of denervation on vocal fold motion. All denervated animals demonstrated immediate right vocal fold paralysis. No obvious vocal cord movement was recorded at any of the time intervals following transection of the RLN. A significant

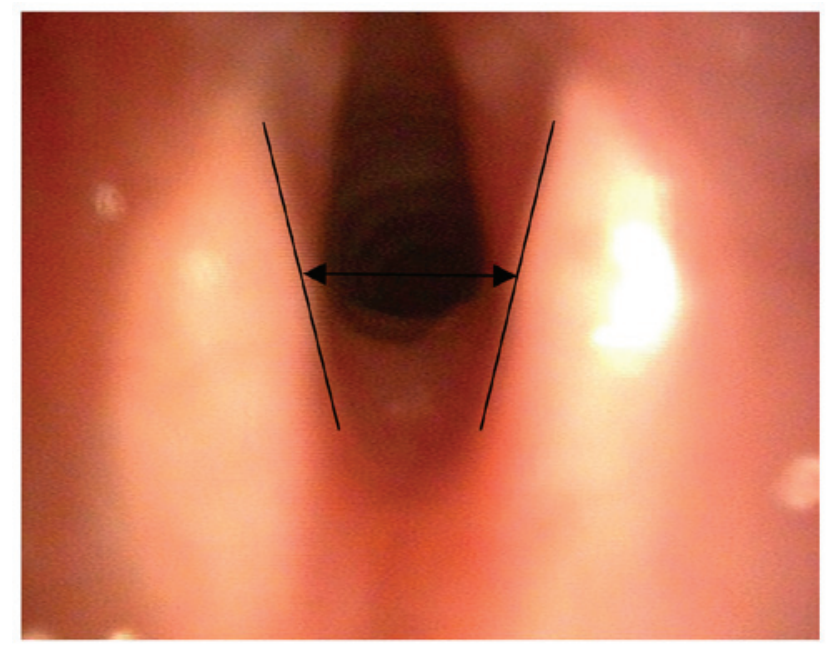

Figure 1. Endoscopic image of the normal rat larynx. Black arrows indicate the angles between the arytenoid cartilages.
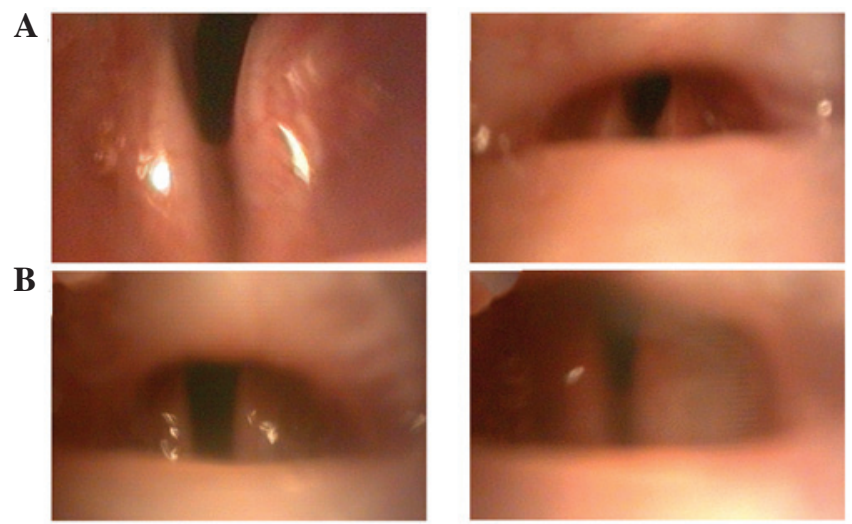

C
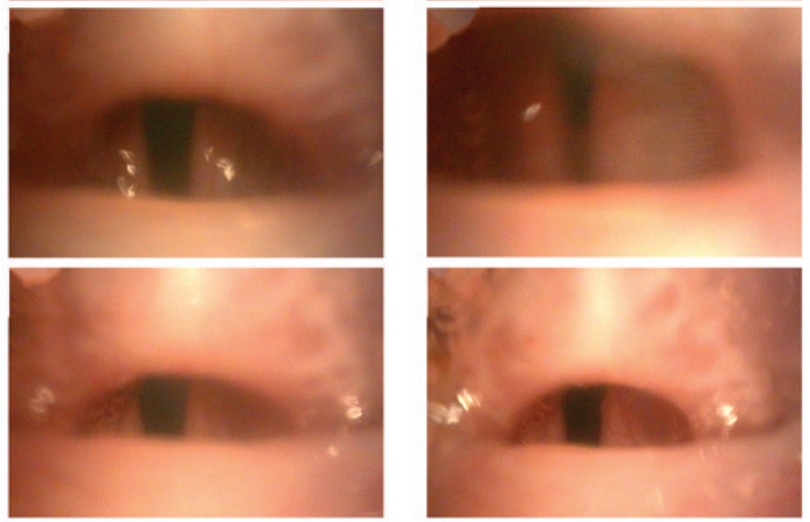

D
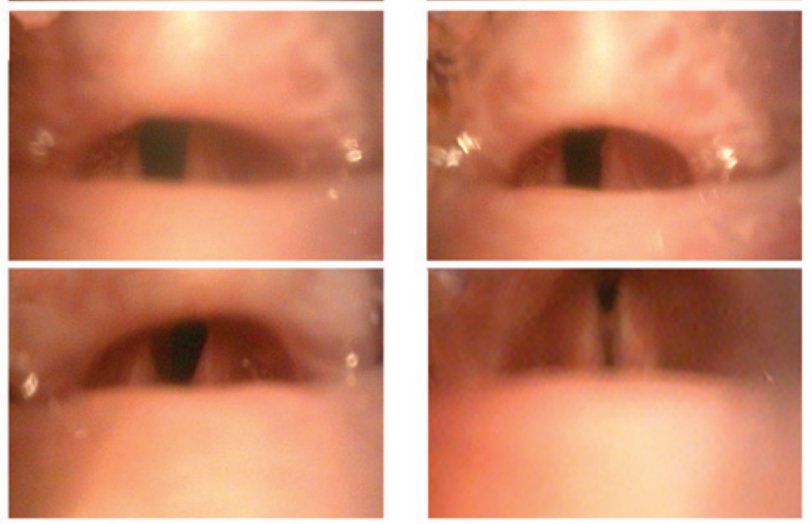

Figure 2. Laryngoscopic image of denervation groups at maximal abduction (left column) and maximal adduction (right column) at (A) 3, (B) 6, (C) 10 and (D) 16 weeks.

difference was indicated for angles between the arytenoid cartilage structures for the denervation and control groups $(\mathrm{P}<0.01)$. The angles of maximal abduction were $21.97 \pm 5.30$, $22.34 \pm 6.79,22.56 \pm 4.94,25.94 \pm 6.92$ and $40.60 \pm 9.36$, at $3,6,10$ and 16 weeks and in the control group, respectively (Fig. 3A), and the maximal adduction angles were $13.14 \pm 5.54^{\circ}$, $11.70 \pm 4.52^{\circ}, 10.29 \pm 3.41^{\circ}, 9.12 \pm 3.99^{\circ}$ and $4.90 \pm 0.22^{\circ}$, at $3,6,10$ and 16 weeks and in the control group, respectively (Fig. 3B). The vocal fold movements on the side of nerve injury did not return to normal during the study. 


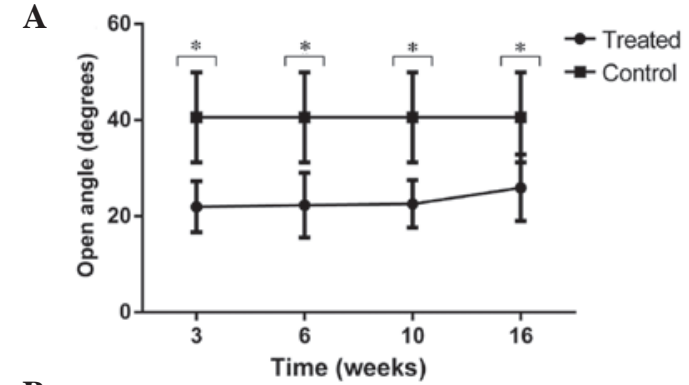

B

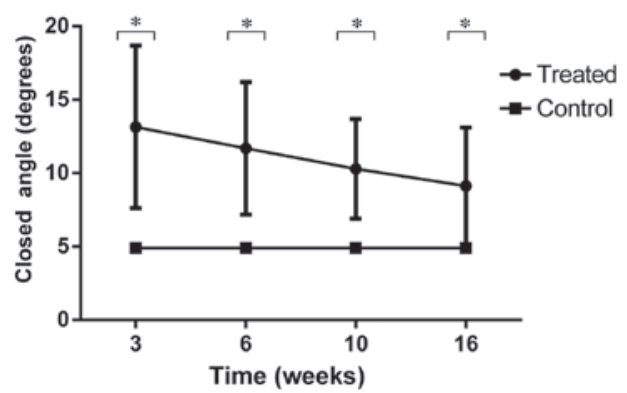

Figure 3. (A) Open and (B) closed angles between arytenoid cartilages during movement of the vocal fold. ${ }^{*} \mathrm{P}<0.05$ vs. the control group.

Effect of denervation on vocalization. The voice spectra of the denervation and control groups are presented in Fig. 4. The vocalization sounded sharp and high-pitched in the control rats, whereas it was hoarse and deep in the denervation groups. The spectral analysis of the control group demonstrated a high amplitude with a wide, continuous waveform (Fig. 4A). Following RLN injury, the spectrum became narrow with a low amplitude. However, the amplitude and width of the continuous waveform increased gradually with time (Fig. 4B-E).

The spectral area (calculated as a percentage) in the denervation group increased gradually over time, with values at 3,6, 10 and 16 weeks of $21.07 \pm 14.41,25.04 \pm 7.50,33.22 \pm 13.89$ and $40.55 \pm 15.36 \%$, respectively (Fig. $5 \mathrm{~A} ; \mathrm{P}<0.01$ vs. the control group). The postoperative amplitudes at $3,6,10$, and 16 weeks were $60.43 \pm 41.49,73.62 \pm 18.72,87.02 \pm 23.40$ and $95.74 \pm 6.38 \%$, respectively (Fig. 5B). Over time, the amplitude percentages increased $(\mathrm{P}<0.05)$, although there was no significant difference between the control group and the 16-week denervation group $(\mathrm{P}=0.0540)$.

Effect of denervation on neurotrophic factor expression. Figs. 6 and 7A present data for the expression of BDNF in the TA and PCA muscles. In the TA muscles, BDNF expression levels decreased slowly over time. However, the expression of BDNF significantly decreased at 3 weeks following injury. Although the expression increased at 6 weeks, it did not reach the baseline level.

Expression of GDNF (Figs. 7B and 8) in the TA was similar to that in the PCA muscle from 3 to 10 weeks; however, at rest, there was a marginal increase in GDNF expression levels in the PCA muscle.

Effect of denervation on RLN histology. Compressed or degenerating axons were observed in certain cases. Fig. 9 demonstrates axons embedded in a fibrous structure. At 16 weeks, the RLN had fewer axons $(\sim 81.00 \pm 7.07$ axons $)$
A

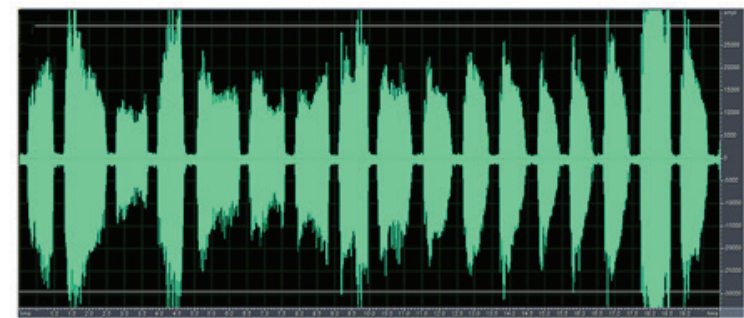

B

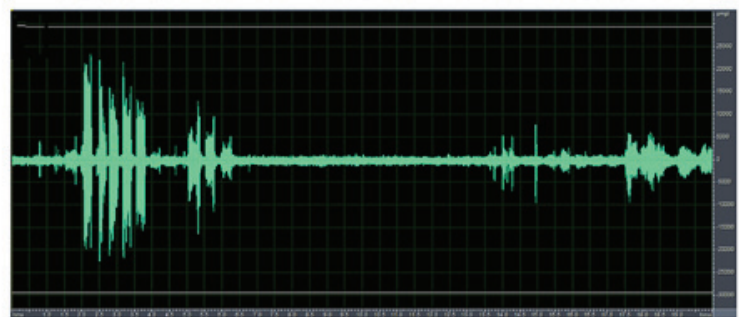

C

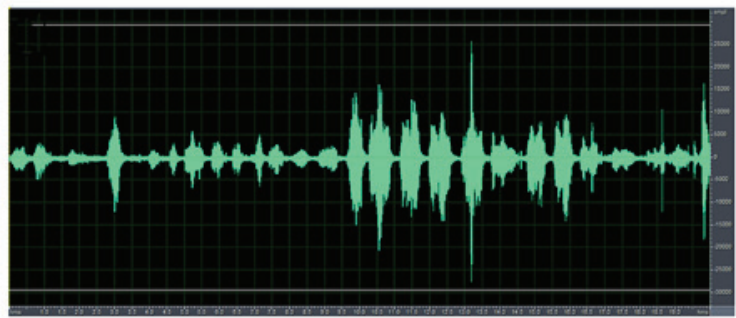

$\mathbf{D}$

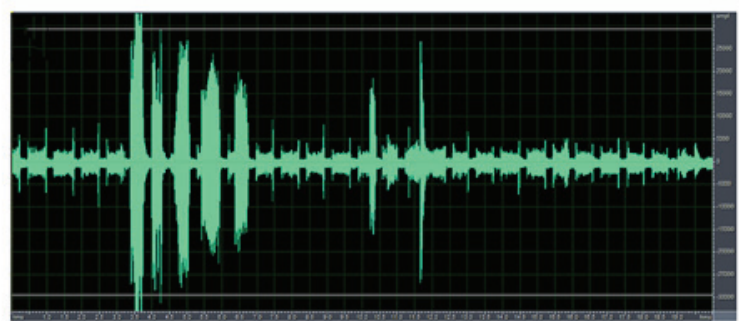

$\mathbf{E}$

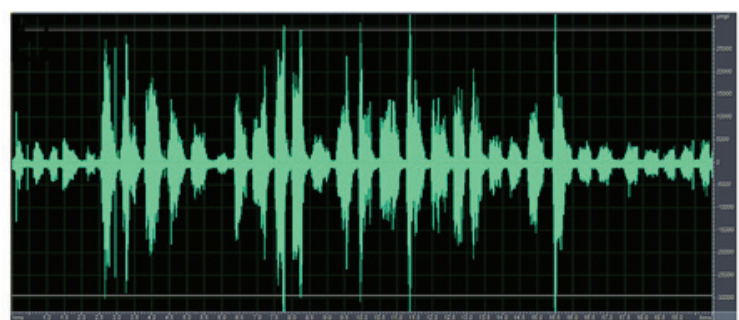

Figure 4. Voice spectrums of (A) the control and denervation groups at (B) 3 , (C) 6, (D) 10 and (E) 16 weeks. The control group exhibits a high amplitude with wide, continuous waveforms, which increase over time.

than the control nerves (Fig. 10). The axon diameters of the RLN sections were recorded. The mean diameter of the axons was $4.38 \pm 1.1 .75 \mu \mathrm{m}$ in normal rats; the right RLN exhibited a reduction for 6 weeks, followed by a slight increase. RLN regrowth was not correlated with vocal fold motion.

\section{Discussion}

In the present study, recovery of vocal fold movement, vocalization, expression levels of BDNF and GDNF, and morphometry of RLN sections were investigated. When the RLN is resected, functional recovery of vocal cord mobility is not achieved. The majority of research studies are limited to examining the return of airway patency, movement of vocal 

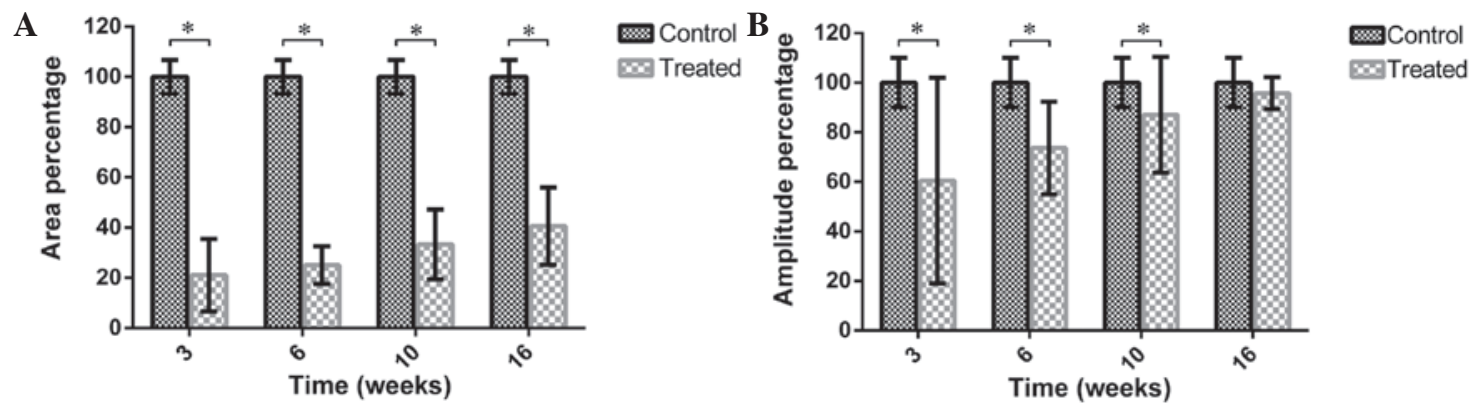

Figure 5. Spectral area and amplitude percentages at different time-points. (A) The spectral area percentage in the denervation group increased gradually over time, however, did not reach the baseline level at any time point following injury. (B) The postoperative amplitude percentages increased gradually over time, and reached the baseline level at 16 weeks. ${ }^{*} \mathrm{P}<0.05$.

A a

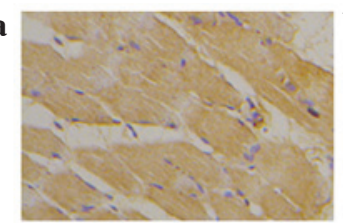

B

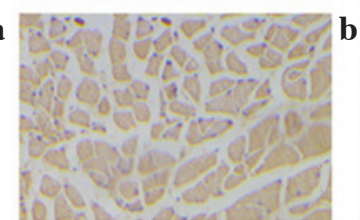

C

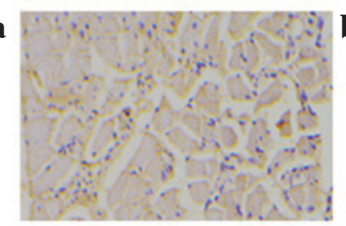

D

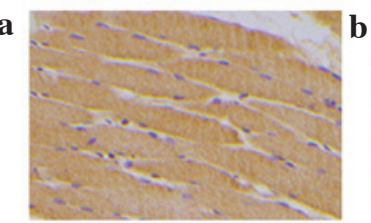

$\mathbf{E}$

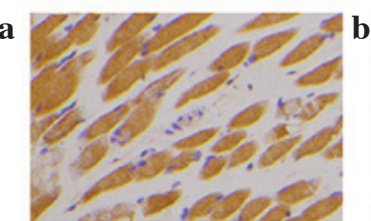

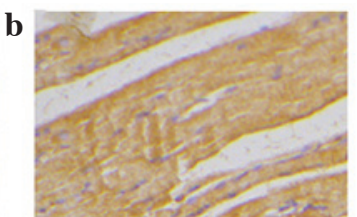
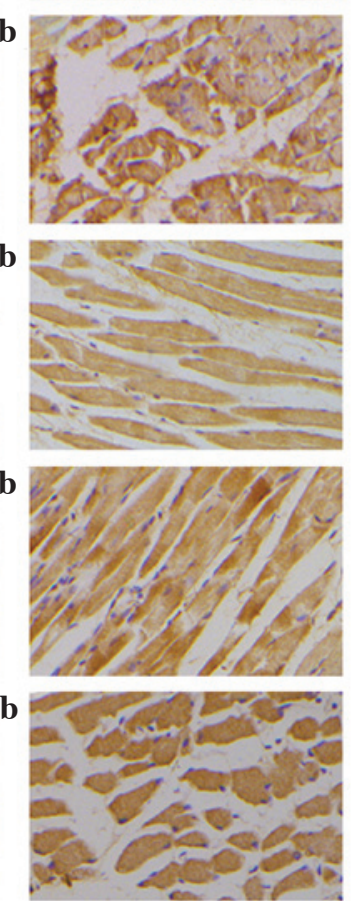
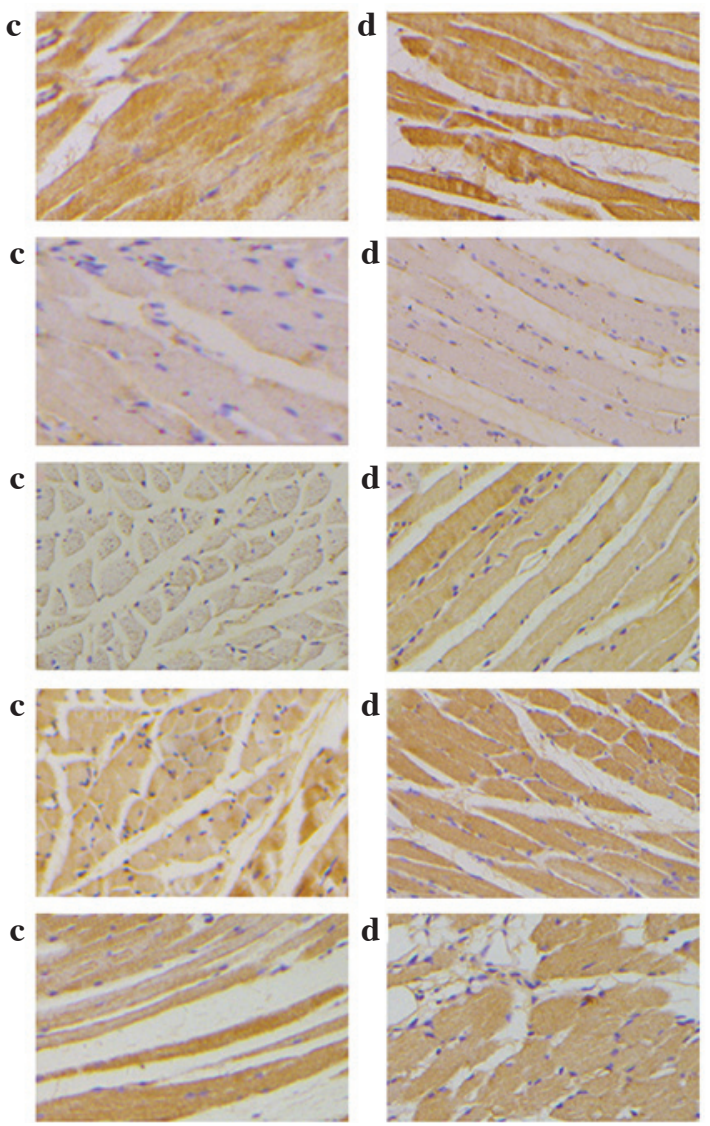

Figure 6. Immunohistochemistry of the (a) right PCA, (b) right TA, (c) left PCA and (d) left TA, stained for brain-derived neurotrophic factor for the (A) control group, and at (B) 3, (C) 6, (D) 10 and (E) 16 weeks. Magnification, x100. PCA, posterior cricoarytenoid; TA, thyroarytenoid.

A

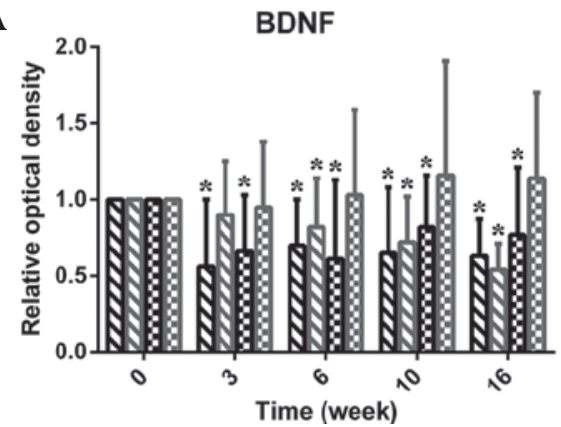

B

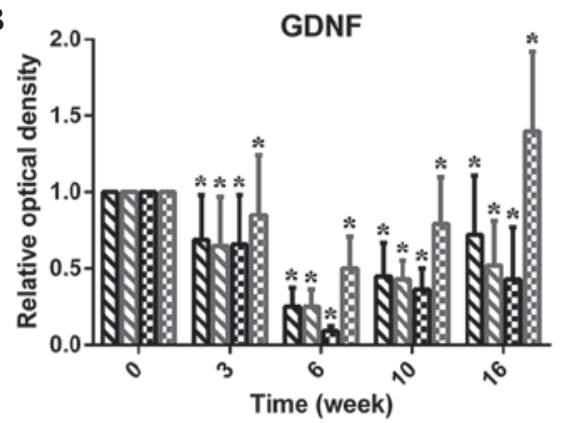

\section{Right PCA Right TA \\ $\otimes$ Left PCA Left TA}

Figure 7. Expression of BDNF and GDNF in TA and PCA muscles. (A) BDNF expression levels were reduced over time in the right TA, and were significantly reduced at 3 weeks following injury in the right PCA. (B) GDNF levels were similar between the TA and PCA muscles from 3-10 weeks. At 16 weeks, the expression levels were greater in the right PCA muscle compared with the right TA muscle. " $\mathrm{P}<0.05$ vs. the control group. BDNF, brain-derived neurotrophic factor; GDNF, glial cell line-derived neurotrophic factor; PCA, posterior cricoarytenoid; TA, thyroarytenoid. 
A a

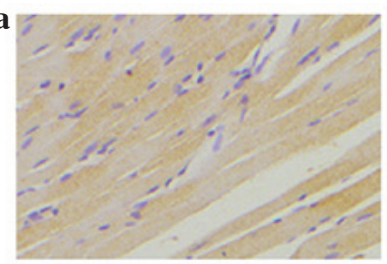

B

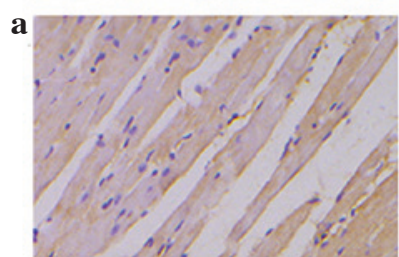

C

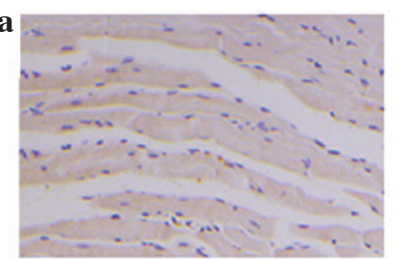

D

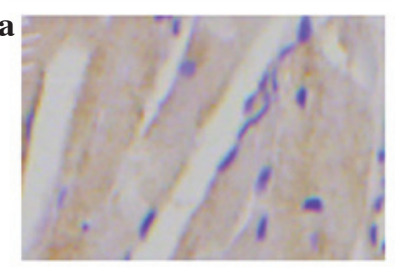

$\mathbf{E}$

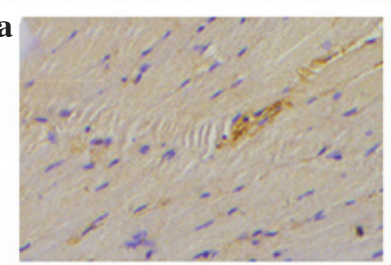

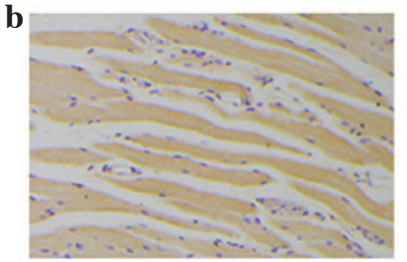
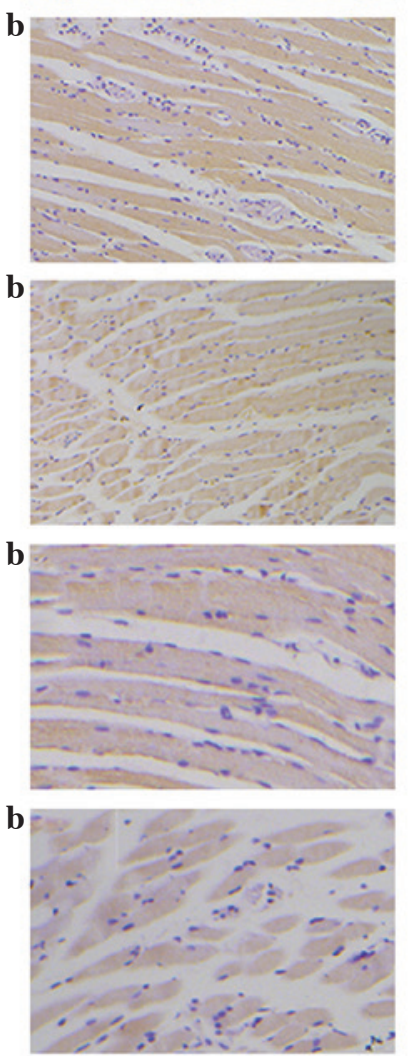

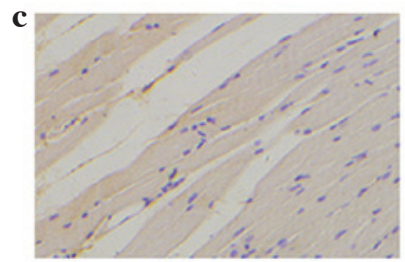

c
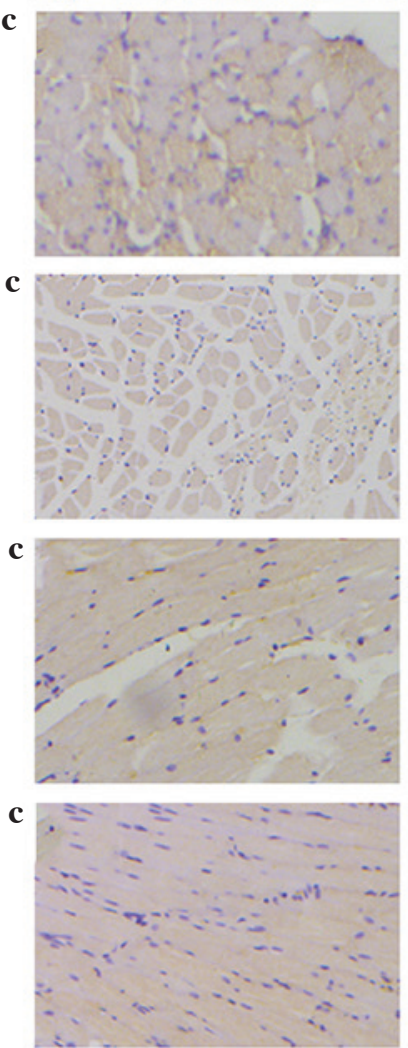
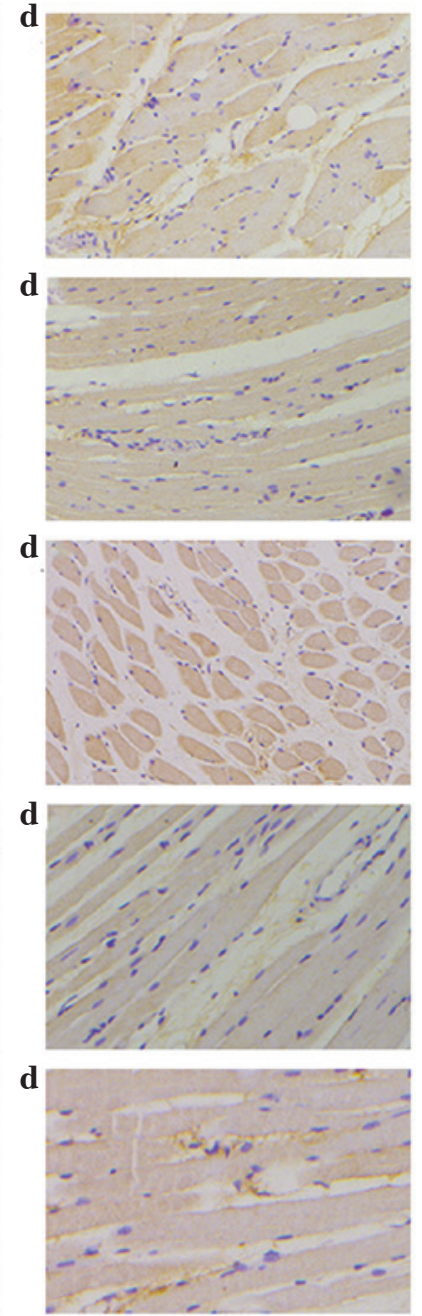

Figure 8. Immunohistochemistry of the (a) right PCA, (b) right TA, (c) left PCA and (d) left TA, stained for glial cell line-derived neurotrophic factor for the (A) control group, and at (B) 3, (C) 6, (D) 10 and (E) 16 weeks. Magnification, x100. PCA, posterior cricoarytenoid; TA, thyroarytenoid.

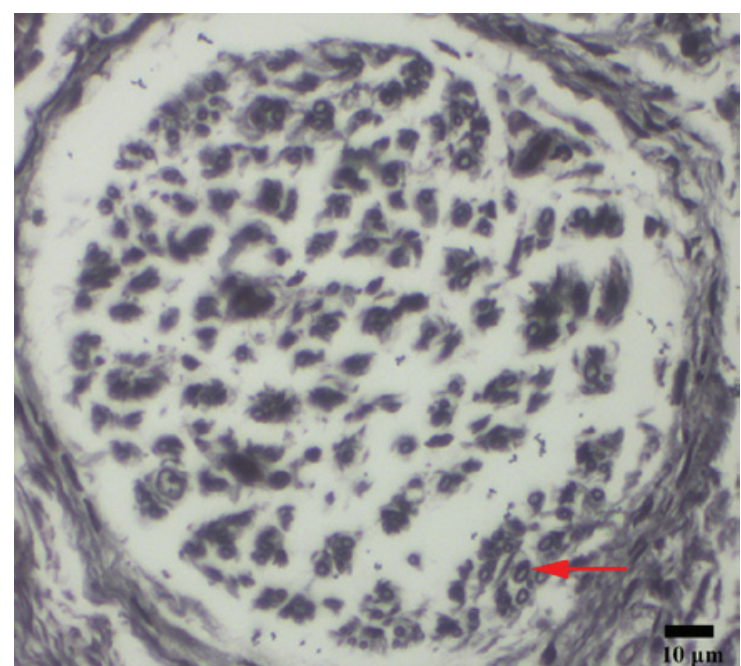

Figure 9. Section of recurrent laryngeal nerve observed by Bielschowsky modification. Red arrow indicates an axon. Scale bar, $10 \mu \mathrm{m}$.

cords or reinnervation. The current study offered a vocalization measurement to reflect the recovery. This method of acoustic wave analysis is original with, to the best of our knowledge, no previous investigations conducted in this manner. The data values were comparable as the same crushing strength was used to stimulate the rats and an equal distance between the microphones and mouths of the rats was maintained; furthermore, the same full-size acoustic window was recorded and equal time was allotted for analysis.

Nerve transection induces Sunderland fifth-degree injury, which involves injury to the nerve trunk (57). Ischemia causes electrolyte concentrations to shift and also results in excessive excitatory amino acid release (58), triggering cytotoxic signaling cascades, myelin destruction and restricted regrowth of axons (17). Transection is a common phenomenon among nerve injuries (59). In the current study, following transection, a degree of functional recovery of vocalization was observed. In addition, the spectral area percentage demonstrated that although voice function had been restored, it did not return to normal levels. The amplitude percentage demonstrated that the rats did not recover to normal levels by 3, 6 and 10 weeks; however they were able to chirp normally at 16 weeks. Although spontaneous reinnervation did not restore the motion of the vocal fold, it may provide improved vocalization compared to complete denervation, perhaps by maintaining the tonus and bulk of the vocal fold (60). 

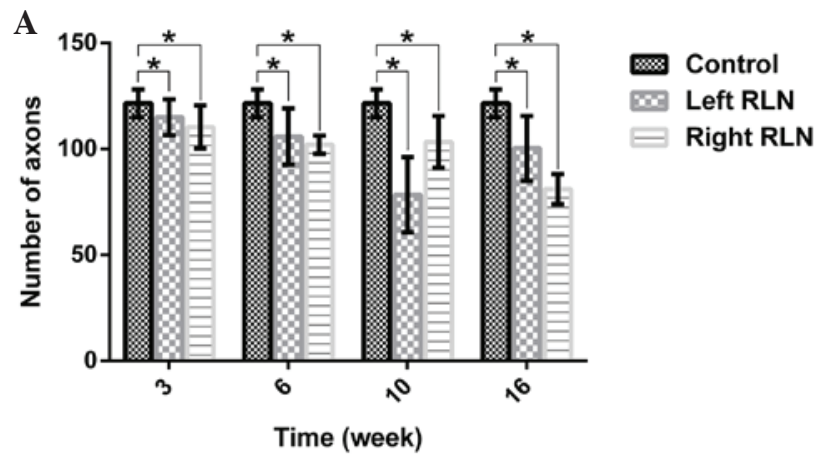

B

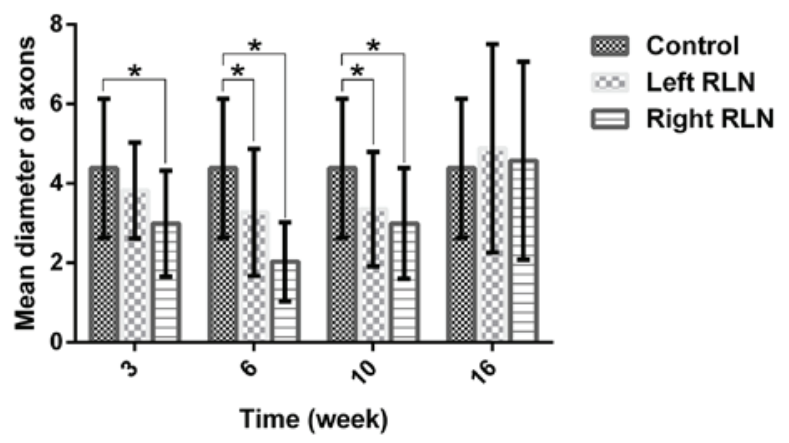

C

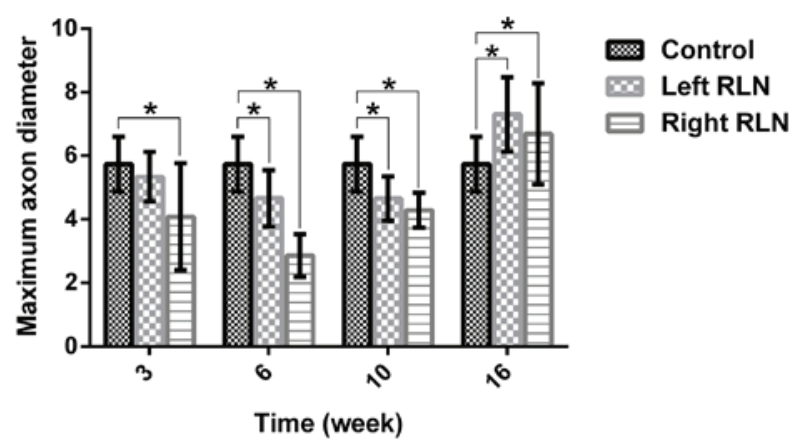

D

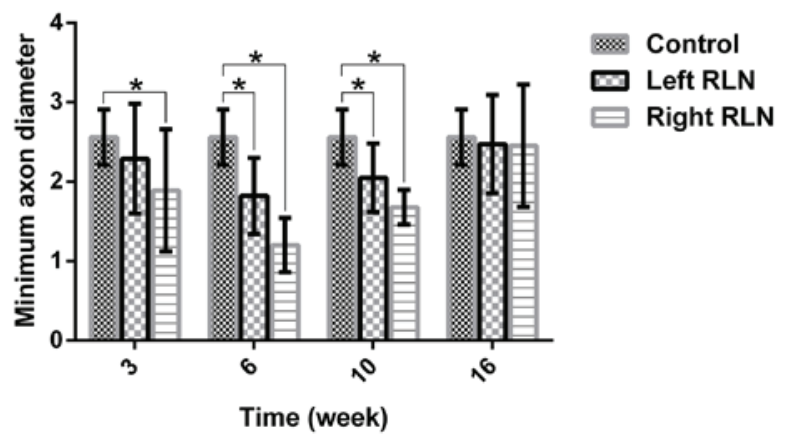

Figure 10. (A) Number and (B-D) diameters of the axons at different time-points. RLN, recurrent laryngeal nerve. ${ }^{*} \mathrm{P}<0.05$.

Following RLN injury, degeneration of axons results in reduced conduction of action potentials. Furthermore, it is well known that axons with a larger diameter have stronger regeneration ability (61). Results from the present study demonstrated that the diameter and number of axons was lower in the denervation group than in the control group. The recovery of axons did not reach the desired level (return to baseline), which may reflect the limited self-repair capability of the axons.

The TA and PCA muscles demonstrated a difference in the expression levels of GDNF; the level of GDNF expression was lower in the denervated muscles than in the control muscles and reached a minimum at 6 weeks. BDNF and GDNF were differentially expressed in the TA and PCA muscles. During the current study, there was no increase in BDNF expression in either the TA or PCA muscles, although large differences in BDNF expression levels were observed between the TA and PCA muscles. In the third week, the level of BDNF expression was reduced markedly in the right PCA muscles, possibly as part of a stress response to the surgery, which triggers catabolism in muscles (62). This requires verification at an earlier time-point. The contralateral TA may have a compensatory role. The differences between the TA and PCA muscles may arise from the various responses to the surgery or may be associated with the type of muscle fibers (19). The TA muscles are primarily comprised of fast-twitch fibers, whereas nearly half of the PCA muscle fibers are slow-twitch (63). Reinnervation of fast-twitch fibers requires fewer muscle fibers than slow-twitch fibers (60). Based on the results of the present study, differences in neurotrophin expression may induce preferential reinnervation of the adductor muscles. This may explain the adducted position of the vocal fold following RLN injury, and may be more plausible than the hypothesis of the present study, that the adducting and abducting forces abrogate each other, resulting in no net motion. Due to the enhancement of glottis closure, the preferential reinnervation of the TA muscle may be favorable in patients with unilateral paralysis, but not in patients with bilateral paralysis. Further studies are required, which focus on changes in other neurotrophins, such as nerve growth factor and neurotrophin-4, and improving PCA muscle function in patients with bilateral paralysis. The horizontal division of the PCA and the superior medial division of the TA are predominantly composed of slow-twitch fibers, whereas the lateral division of the TA, and the oblique and vertical divisions of the PCA have a higher percentage of fast-twitch fibers; therefore, future studies are required to differentiate between the muscle types (64). Due to the specific functions of slow- and fast-twitch muscle fibers, the expression of BDNF and GDNF is different (22). It may therefore be possible to apply neurotrophic factors to promote nerve regeneration by compensating for the reduction in their levels following injury.

The present study has certain limitations. The study was conducted over 16 weeks in a small number of rats; therefore, large-scale, short- and long-term studies are required to confirm the results. In addition, the lack of vocal fold motion may have been due to synkinetic reinnervation or failed reinnervation of the laryngeal muscles. Furthermore, the respiratory cycle of the animals should be monitored to determine the reason for the lack of vocal fold motion. Finally, immunohistochemistry was used to observe the expression of GDNF and BDNF proteins within the muscles. Although this approach is valuable for localizing specific proteins in tissues, studies supported by quantitative techniques, such as western blotting, are required to confirm and expand the results (19).

The results of the present study demonstrate that the restoration of the vocal fold is not significant, despite spontaneous regeneration. In humans, the spontaneous regeneration of the RLN remains a controversial subject $(7,60,65)$. Spontaneous laryngeal reinnervation may occur through regeneration of a healthy residual RLN or from nerves in the surrounding area (3). Strategies to promote sufficient reinnervation of the 
PCA muscle may improve the effectiveness of spontaneous recovery following RLN injury.

In conclusion, although only vocal function improved during the present study, spontaneous reinnervation was observed following RLN injury. Thus, neurotrophic factors may be utilized following RLN injury to promote repair of neurological function.

\section{Acknowledgements}

The present study was supported by a grant from the National Natural Science Foundation of China (grant no. 81271067).

\section{References}

1.Zhang N, Yan $\mathrm{H}$ and Wen X: Tissue-engineering approaches for axonal guidance. Brain Res Brain Res Rev 49: 48-64, 2005.

2. Kingham PJ and Terenghi G: Bioengineered nerve regeneration and muscle reinnervation. J Anat 209: 511-526, 2006.

3. Dalgic A, Kandogan T, Koc M, Kulan CA, Yagci A, Engin O, Aksoy G and Ozuer MZ: Short-term laryngeal electromyography and histopathological findings after primary reconstruction of the inferior laryngeal nerve in rabbits: Prospective study. J Laryngol Otol 127: 48-53, 2013.

4. Moro K, Shiotani A, Watabe K, Takeda Y, Saito K, Mori Y and Ogawa K: Adenoviral gene transfer of BDNF and GDNF synergistically prevent motoneuron loss in the nucleus ambiguus. Brain Res 1076: 1-8, 2006.

5. Caldarelli DD and Holinger LD: Complications and sequelae of thyroid surgery. Otolaryngol Clin North Am 13: 85-97, 1980.

6. Tessema B, Pitman MJ, Roark RM, Berzofsky C, Sharma S and Schaefer SD: Evaluation of functional recovery of recurrent laryngeal nerve using transoral laryngeal bipolar electromyography: A rat model. Ann Otol Rhinol Laryngol 117: 604-608, 2008.

7. Crumley RL and McCabe BF: Regeneration of the recurrent laryngeal nerve. Otolaryngol Head Neck Surg 90: 442-447, 1982

8. Shindo ML, Herzon GD, Hanson DG, Cain DJ and Sahgal V: Effects of denervation on laryngeal muscles: A canine model. Laryngoscope 102: 663-669, 1992.

9. Horsley JS: Suture of the Recurrent Laryngeal Nerve. Ann Surg 52: 287-288, 1910.

10. Gordon JH and McCabe BF: The effect of accurate neurorrhaphy on reinnervation and return of laryngeal function. Laryngoscope 78: 236-250, 1968.

11. Crumley RL: Laryngeal synkinesis revisited. Ann Otol Rhinol Laryngol 109: 365-371, 2000.

12. Flint PW, Downs DH and Coltrera MD: Laryngeal synkinesis following reinnervation in the rat. Neuroanatomic and physiologic study using retrograde fluorescent tracers and electromyography. Ann Otol Rhinol Laryngol 100: 797-806, 1991.

13. Tessema B, Roark RM, Pitman MJ, Weissbrod P, Sharma S and Schaefer SD: Observations of recurrent laryngeal nerve injury and recovery using a rat model. Laryngoscope 119: 1644-1651, 2009.

14. Woodson GE: Spontaneous laryngeal reinnervation after recurrent laryngeal or vagus nerve injury. Ann Otol Rhinol Laryngol 116: 57-65, 2007.

15. Miyamaru S, Kumai Y, Ito T and Yumoto E: Effects of long-term denervation on the rat thyroarytenoid muscle. Laryngoscope 118: 1318-1323, 2008

16. Choi JS, Oh SH, An HY, Kim YM, Lee JH and Lim JY: Functional regeneration of recurrent laryngeal nerve injury during thyroid surgery using an asymmetrically porous nerve guide conduit in an animal model. Thyroid 24: 52-59, 2014.

17. Araki K, Shiotani A, Watabe K, Saito K, Moro K and Ogawa K: Adenoviral GDNF gene transfer enhances neurofunctional recovery after recurrent laryngeal nerve injury. Gene Ther 13 296-303, 2006.

18. Meller SM: Functional anatomy of the larynx. Otolaryngol Clin North Am 17: 3-12, 1984.

19. Vega-Cordova X, Cosenza NM, Helfert RH and Woodson GE: Neurotrophin expression of laryngeal muscles in response to recurrent laryngeal nerve transection. Laryngoscope 120: $1591-1596,2010$.
20. Kupfer RA, Old MO, Oh SS, Feldman EL and Hogikyan ND: Spontaneous laryngeal reinnervation following chronic recurrent laryngeal nerve injury. Laryngoscope 123: 2216-2227, 2013.

21. Kumai Y, Ito T, Matsukawa A and Yumoto E: Effects of denervation on neuromuscular junctions in the thyroarytenoid muscle. Laryngoscope 115: 1869-1872, 2005.

22. Halum SL, Bijangi-Vishehsaraei K, Saadatzadeh MR and McRae BR: Differences in laryngeal neurotrophic factor gene expression after recurrent laryngeal nerve and vagus nerve injuries. Ann Otol Rhinol Laryngol 122: 653-663, 2013.

23. Crumley RL: Update: Ansa cervicalis to recurrent laryngeal nerve anastomosis for unilateral laryngeal paralysis. Laryngoscope 101: 384-387, 1991.

24. Zheng H, Li Z, Zhou S, Cuan Y and Wen W: Update: Laryngeal reinnervation for unilateral vocal cord paralysis with the ansa cervicalis. Laryngoscope 106: 1522-1527, 1996.

25. Lee WT, Milstein C, Hicks D, Akst LM and Esclamado RM: Results of ansa to recurrent laryngeal nerve reinnervation. Otolaryngol Head Neck Surg 136: 450-454, 2007.

26. Unuma K, Chen J, Saito S, Kobayashi N, Sato K, Saito K, Wakisaka H, Mominoki K, Sano A and Matsuda S: Changes in expression of prosaposin in the rat facial nerve nucleus after facial nerve transection. Neurosci Res 52: 220-227, 2005.

27. Li J and Shi R: A device for the electrophysiological recording of peripheral nerves in response to stretch. J Neurosci Methods 154: 102-108, 2006.

28. Tun K, Cemil B, Gurcay AG, Kaptanoglu E, Sargon MF, Tekdemir I, Comert A and Kanpolat Y: Ultrastructural evaluation of Pulsed Radiofrequency and Conventional Radiofrequency lesions in rat sciatic nerve. Surg Neurol 72: 496-501, 2009.

29. Zelano J, Plantman S, Hailer NP and Cullheim S: Altered expression of nectin-like adhesion molecules in the peripheral nerve after sciatic nerve transection. Neurosci Lett 449: 28-33, 2009.

30. Yayama T, Kobayashi S, Nakanishi Y, Uchida K, Kokubo Y, Miyazaki T, Takeno K, Awara K, Mwaka ES, Iwamoto Y and Baba H: Effects of graded mechanical compression of rabbit sciatic nerve on nerve blood flow and electrophysiological properties. J Clin Neurosci 17: 501-505, 2010.

31. Maarrawi J, Kobaiter-Maarrawi S, Ghanem I, Ali Y, Aftimos G, Okais N and Samaha E: Pathological effects and motor response threshold changes following radiofrequency application at various distances from the L-5 nerve root: An experimental study. J Neurosurg Spine 15: 285-291, 2011.

32. Rickett T, Connell S, Bastijanic J, Hegde S and Shi R: Functional and mechanical evaluation of nerve stretch injury. J Med Syst 35: 787-793, 2011.

33. Sacharuk VZ, Lovatel GA, Ilha J, Marcuzzo S, Pinho AS, Xavier LL, Zaro MA and Achaval M: Thermographic evaluation of hind paw skin temperature and functional recovery of locomotion after sciatic nerve crush in rats. Clinics (Sao Paulo) 66: 1259-1266, 2011

34. Wang Y, Tang P, Zhang L, Wan W, He C and Tang J: Gray-scale contrast-enhanced ultrasonography for quantitative evaluation of the blood perfusion of the sciatic nerves with crush injury. Acad Radiol 18: 1285-1291, 2011.

35. Zhu L, Yan Y, Ke K, Wu X, Gao Y, Shen A, Li J, Kang L, Zhang G, Wu Q and Yang H: Dynamic change of Numbl expression after sciatic nerve crush and its role in Schwann cell differentiation. J Neurosci Res 90: 1557-1565, 2012.

36. English AW, Liu K, Nicolini JM, Mulligan AM and Ye K: Small-molecule trkB agonists promote axon regeneration in cut peripheral nerves. Proc Natl Acad Sci USA 110: 16217-16222, 2013.

37. Kaya Y, Sarıkcığlu L, Aslan M, Kencebay C, Demir N, Derin N, Angelov DN and Yıldırım FB: Comparison of the beneficial effect of melatonin on recovery after cut and crush sciatic nerve injury: A combined study using functional, electrophysiological, biochemical, and electron microscopic analyses. Childs Nerv Syst 29: 389-401, 2013.

38. Li S, Liu Q, Wang Y, Gu Y, Liu D, Wang C, Ding G, Chen J, Liu J and $\mathrm{Gu} \mathrm{X}$ : Differential gene expression profiling and biological process analysis in proximal nerve segments after sciatic nerve transection. PLoS One 8: e57000, 2013.

39. Schmid AB, Coppieters MW, Ruitenberg MJ and McLachlan EM: Local and remote immune-mediated inflammation after mild peripheral nerve compression in rats. J Neuropathol Exp Neurol 72: 662-680, 2013. 
40. Singleton JR, Dixit VM and Feldman EL: Type I insulin-like growth factor receptor activation regulates apoptotic proteins. J Biol Chem 271: 31791-31794, 1996.

41. Kim B, Leventhal PS, Saltiel AR and Feldman EL: Insulin-like growth factor-I-mediated neurite outgrowth in vitro requires mitogen-activated protein kinase activation. J Biol Chem 272: 21268-21273, 1997.

42. Russell JW, Windebank AJ, Schenone A and Feldman EL: Insulin-like growth factor-I prevents apoptosis in neurons after nerve growth factor withdrawal. J Neurobiol 36: 455-467, 1998.

43. Baumgartner BJ and Shine HD: Targeted transduction of CNS neurons with adenoviral vectors carrying neurotrophic factor genes confers neuroprotection that exceeds the transduced population. J Neurosci 17: 6504-6511, 1997.

44. Giménez y Ribotta M, Revah F, Pradier L, Loquet I, Mallet J and Privat A: Prevention of motoneuron death by adenovirus-mediated neurotrophic factors. J Neurosci Res 48: 281-285, 1997.

45. Dittrich F, Thoenen H and Sendtner M: Ciliary neurotrophic factor: Pharmacokinetics and acute-phase response in rat. Ann Neurol 35: 151-163, 1994.

46. Halum SL, McRae B, Bijangi-Vishehsaraei K and Hiatt K Neurotrophic factor-secreting autologous muscle stem cell therapy for the treatment of laryngeal denervation injury. Laryngoscope 122: 2482-2496, 2012.

47. Heavner SB, Rubin AD, Fung K, Old M, Hogikyan ND and Feldman EL: Dysfunction of the recurrent laryngeal nerve and the potential of gene therapy. Ann Otol Rhinol Laryngol 116 441-448, 2007.

48. Kingham PJ, Hughes A, Mitchard L, Burt R, Murison P, Jones A Terenghi $G$ and Birchall MA: Effect of neurotrophin-3 on reinnervation of the larynx using the phrenic nerve transfer technique. Eur J Neurosci 25: 331-340, 2007.

49. Saito K, Shiotani A, Watabe K, Moro K, Fukuda H and Ogawa K Adenoviral GDNF gene transfer prevents motoneuron loss in the nucleus ambiguus. Brain Res 962: 61-67, 2003.

50. Shiotani A, Saito K, Araki K, Moro K and Watabe K: Gene therapy for laryngeal paralysis. Ann Otol Rhinol Laryngol 116: $115-122,2007$.

51. Zheng H, Zhou S and You Z: The expression and distribution of ciliary neurotrophic factor in laryngeal nerve regeneration. Zhonghua Er Bi Yan Hou Ke Za Zhi 34: 289-292, 1999 (In Chinese).

52. Aoyama T, Kumai Y, Yumoto E, Ito T and Miyamaru S: Effects of nerve-muscle pedicle on immobile rat vocal folds in the presence of partial innervation. Ann Otol Rhinol Laryngol 119: $823-829,2010$
53. Pitman MJ, Weissbrod P, Roark R, Sharma S and Schaefer SD: Electromyographic and histologic evolution of the recurrent laryngeal nerve from transection and anastomosis to mature reinnervation. Laryngoscope 121: 325-331, 2011.

54. Hernández-Morato I, Valderrama-Canales FJ, Berdugo G, Arias G, McHanwell S, Sañudo J, Vásquez T and Pascual-Font A: Reorganization of laryngeal motoneurons after crush injury in the recurrent laryngeal nerve of the rat. J Anat 222: 451-461, 2013.

55. Iizuka T: Experimental studies on the nerve interception and atrophy of the intrinsic muscles of the larynx. J Otolaryng Jap 69: 176-195, 1966 (In Japanese).

56. Owens CM, Marga F, Forgacs G and Heesch CM: Biofabrication and testing of a fully cellular nerve graft. Biofabrication 5: 045007, 2013.

57. Sunderland S: A classification of peripheral nerve injuries producing loss of function. Brain 74: 491-516, 1951.

58. Carlson SL, Parrish ME, Springer JE, Doty K and Dossett L: Acute inflammatory response in spinal cord following impact injury. Exp Neurol 151: 77-88, 1998.

59. Zábrodský M, Bouček J, Kastner J, Kuchař M, Chovanec M and Betka J: Immediate revision in patients with bilateral recurrent laryngeal nerve palsy after thyroid and parathyroid surgery. How worthy is it? Acta Otorhinolaryngol Ital 32: 222-228, 2012

60. Blitzer A, Jahn AF and Keidar A: Semon's law revisited: An electromyographic analysis of laryngeal synkinesis. Ann Otol Rhinol Laryngol 105: 764-769, 1996.

61. Toya Y, Kumai Y, Minoda R and Yumoto E: Modulation of nerve fibers in the rat thyroarytenoid muscle following recurrent laryngeal nerve injury. Acta Otolaryngol 132: 305-313, 2012.

62. Tetzlaff J, Tanzer L and Jones KJ: Exogenous androgen treatment delays the stress response following hamster facial nerve injury. J Neuroendocrinol 19: 383-389, 2007.

63. Vega-Cordova X, Cosenza NM, Helfert RH and Woodson GE: Neurotrophin expression of laryngeal muscles in response to recurrent laryngeal nerve transection. Laryngoscope 120: 1591-1596, 2010

64. Zealear DL and Billante CR: Neurophysiology of vocal fold paralysis. Otolaryngol Clin North Am 37: 1-23, 2004.

65. Damrose EJ, Huang RY, Blumin JH, Blackwell KE, Sercarz JA and Berke GS: Lack of evoked laryngeal electromyography response in patients with a clinical diagnosis of vocal cord paralysis. Ann Otol Rhinol Laryngol 110: 815-819, 2001. 